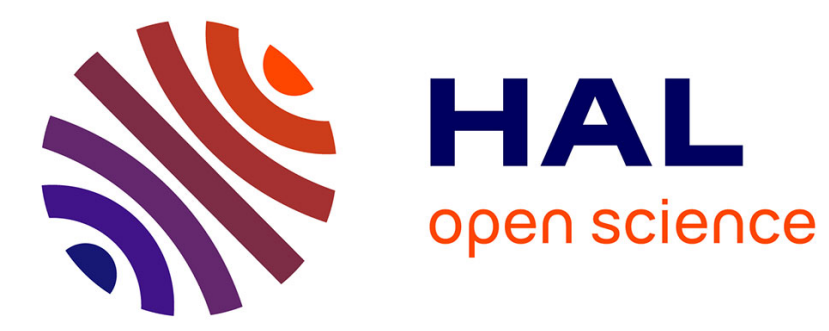

\title{
Specific Surface Area Model for Foam Permeability
}

Olivier Pitois, Elise Lorenceau, N. Louvet, Florence Rouyer

\section{To cite this version:}

Olivier Pitois, Elise Lorenceau, N. Louvet, Florence Rouyer. Specific Surface Area Model for Foam Permeability. Langmuir, 2009, 25, pp.97-100. 10.1021/la8029616 . hal-00507015

\section{HAL Id: hal-00507015 https://hal.science/hal-00507015}

Submitted on 10 Sep 2015

HAL is a multi-disciplinary open access archive for the deposit and dissemination of scientific research documents, whether they are published or not. The documents may come from teaching and research institutions in France or abroad, or from public or private research centers.
L'archive ouverte pluridisciplinaire HAL, est destinée au dépôt et à la diffusion de documents scientifiques de niveau recherche, publiés ou non, émanant des établissements d'enseignement et de recherche français ou étrangers, des laboratoires publics ou privés. 


\section{Specific surface area model for foam permeability}

O. Pitois ${ }^{*}$, E. Lorenceau, N. Louvet and F. Rouyer, Université Paris-Est, Laboratoire de Physique des Matériaux Divisés et des Interfaces, UMR CNRS 8108, 5 bvd Descartes, 77454 Marne la Vallée Cedex 2, France.

* Corresponding author: olivier.pitois@univ-mlv.fr

$\mathrm{Ph}: 33160957324$

Fax : 33160957297

ABSTRACT: Liquid foams have been early recognized as porous materials, the liquid flowing between the gas bubbles. Drainage theories have been established and foams permeability has been modelled from the microscopic description of the equivalent pores geometry, emphasizing similarities with their solid counterparts. But to what extent the theoretical work devoted to the permeability of solid porous materials can be useful to liquid foams ? In this paper, the applicability of the Carman-Kozeny model on foam is investigated. We performed measurements for the permeability of foams with non-mobile surfactants and we show that introducing an equivalent specific surface area for the foam, the model accurately describes the experimental data over two orders of magnitude for the foam liquid fraction, without any additional parameter. Finally, it is shown that this model includes the previous permeability models derived for foams in the dry foams limit.

KEYWORDS: permeability, Carman-Kozeny, porous media, drainage, protein foam 
Toc graphic

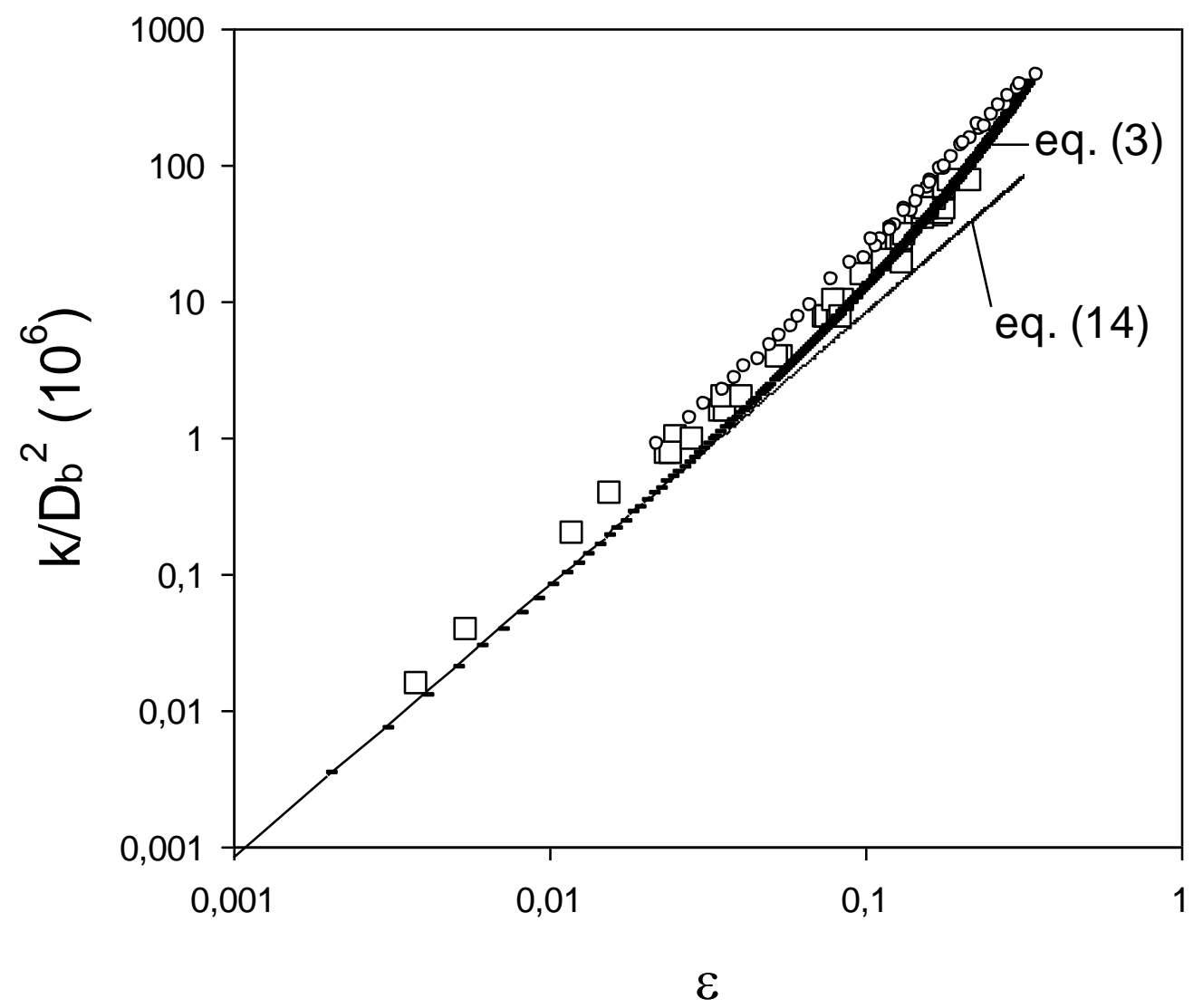




\section{INTRODUCTION}

The Darcy law is an empirical equation based on measurements of the flow of water through sands ans sand-stones [1]. By means of the permeability $\mathrm{k}$, it relates the superficial liquid velocity to the pressure gradient responsible for the flow:

$$
\vec{u}=-\frac{k}{\mu} \overrightarrow{\nabla P}
$$

where $\mu$ is the bulk shear viscosity of the flowing fluid. $k$ quantitatively describes the ability of the solid porous material to let fluids flow through it.

Although composed of fluids, liquid foams have been treated as porous materials, evaluating their permeability with respect to the liquid that can flow between the gas bubbles, and several descriptions for the foam permeability have been proposed [2-7]. But to what extent the theoretical work devoted to the permeability of porous materials can be useful to liquid foams ? Several response elements are presented in the following.

\section{CARMAN-KOZENY MODEL FOR THE FLOW THROUGH GRANULAR BEDS}

Kozeny [8] and Carman [9] proposed to extend the laws of liquid flow through pipes to porous materials. Within this theoretical framework, the porosity of the material is assumed to be composed of a series of channels of the same cross-section. A relevant parameter has been found to be the mean hydraulic radius of the pipes, that is: $m$, the ratio of the volume of fluid in pipes over the surface presented to fluid. Equivalently, $m$ can be expressed using $S_{p}$, the surface area of the porous material (presented to fluid), and $V_{t}$ the total volume of material: $m=\varepsilon V_{t} / S_{p}$, where $\varepsilon$ is the porosity. The ratio $S_{p} / V_{t}$ is called the specific surface area of the porous material: 


$$
A_{S}=\frac{S_{p}}{V_{t}}=\frac{\varepsilon}{m}
$$

Within this channels model, eq.(2) relates the geometrical description of the material on the microscopic scale to macroscopic quantities. From $A_{s}$, an expression for the permeability of the material has been derived [9]:

$$
k=\frac{\varepsilon^{3}}{C_{K} A_{S}^{2}}
$$

Eq. (3) is known as the Carman-Kozeny equation and $C_{K}$ is the Kozeny constant; it is supposed to be of a few units and almost constant for a large variety of conditions. This is generally verified from comparison of eq.(3) with results of experimental and numerical studies performed on granular beds $[10,11]$. For sphere packings, the value $C_{K} \approx 5$ is widely accepted [12]. The use of eq.(3) is particularly convenient because the problem of calculating $\mathrm{k}$ reduces to determining $\mathrm{A}_{\mathrm{s}}$ and $\varepsilon$. Both of these quantities can be measured from imaging procedures of the porous medium or adsorption methods [12].

\section{EQUIVALENT SPECIFIC SURFACE AREA AND PERMEABILITY OF LIQUID FOAMS}

At equilibrium, the structure of liquid foams results from the minimisation of the interfacial energy and can be assessed using a dedicated software, such as Surface Evolver [13]. For the Kelvin foam structure, numerical data are now available, and in the following, we show that a precise value of an equivalent specific surface area can be deduced from these data.

As it is generally admitted that the liquid films have a negligible contribution to liquid drainage, it is useful to introduce $S_{f}$, the interfacial area involved in the foam films, and $S$, 
the total interfacial area of the foam. We assume that the surface area presented to liquid flow is obtained from the following relation:

$$
S_{p}(\varepsilon)=S(\varepsilon)-S_{f}(\varepsilon)
$$

The quantity $S / V_{t}$ can be expressed as:

$$
\frac{S(\varepsilon)}{V_{t}}=\frac{3}{R}(1-\varepsilon) \frac{s(\varepsilon)}{s_{0}}
$$

where $s(\varepsilon) / s_{0}$ is the surface area of a bubble normalized by the surface of the volumeequivalent spherical bubble. Introducing the dimensionless excess energy density $U(\varepsilon)[14,15]$, one can write:

$$
\frac{s(\varepsilon)}{s_{0}}=1+\frac{U}{3(1-\varepsilon)}
$$

A numerical evaluation of $U(\varepsilon)$ for the Kelvin foam has been recently presented in [15] and the corresponding values are used to plot $s(\varepsilon) / s_{0}$ as a function of $\varepsilon$ in figure 1 (the published data for $U(\varepsilon)[15]$ have been extrapolated from $\varepsilon=0.25$ to $\varepsilon=0.32)$. The bubble surface area is a decreasing function of the liquid volume fraction until the limit value $\varepsilon=0.32$, corresponding to the BCC structure for the assembly of spherical bubbles, is reached. Above $\varepsilon$ $=0.32$, the bubbles do not touch each other, thus $S_{f}=0$ and $S$ is constant. For $\varepsilon=0$, $s_{\varepsilon=0}=C s_{0}$ is the bubble surface area in the dry limit for the Kelvin foam, with $C=$ $27 / 4 \pi(6 \sqrt{2} / \pi)^{2 / 3} \approx 1.1$ 


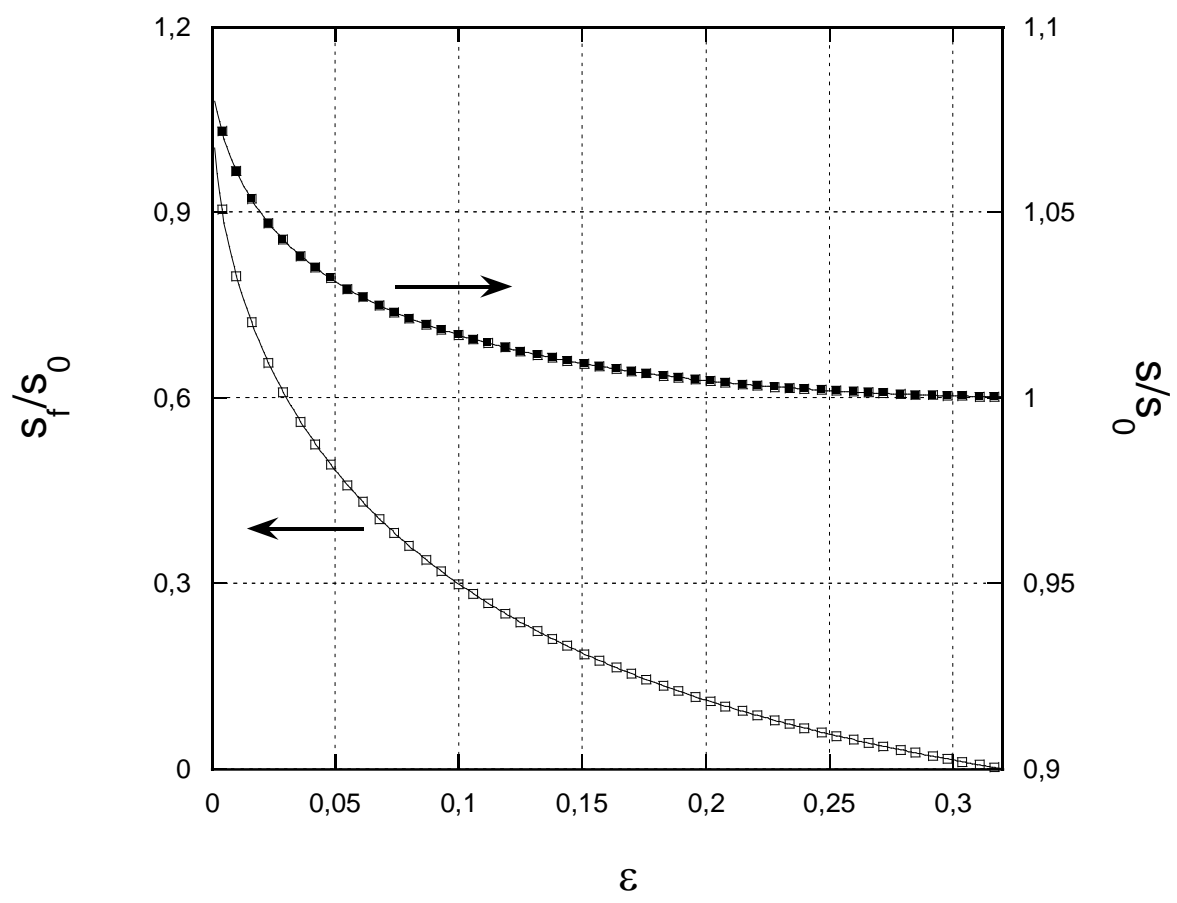

Figure 1: Surface area of a bubble $(S)$ and films surface area of this bubble $\left(S_{f}\right)$ normalized by the surface of the volume-equivalent spherical bubble $\left(S_{0}\right)$ as a function of $\varepsilon$.

We define the fraction of the total surface area of a bubble covered by thin films as:

$$
\alpha(\varepsilon)=s_{f} / s_{\varepsilon=0}
$$

where $s_{f}$ is the surface area covered by the films. An analytical approximate expression for $\alpha(\varepsilon)$ has been provided by Hilgenfeldt et al. [16]:

$$
\alpha(\varepsilon) \approx\left(1-1.52 \varepsilon^{1 / 2}\right)^{2}
$$


As eq.(8) was given to be valid up to $\varepsilon \approx 0.2[16], s_{f} / s_{\varepsilon=0}$ has been extrapolated over the range $0.2 \leq \varepsilon \leq 0.32$, ensuring $s_{f} / s_{\varepsilon=0}=0$ for $\varepsilon=0.32$ (see figure 1 ).

In the foam, the total interfacial area corresponding to the films is:

$$
\frac{S_{f}}{V_{t}}=\frac{3}{R}(1-\varepsilon) \frac{S_{f}}{S_{0}}
$$

where $s_{f} / s_{0}=C \alpha(\varepsilon)$ is the films surface area of a bubble normalized by the surface of the volume-equivalent spherical bubble and is plotted in figure 1 as a function of $\varepsilon$.

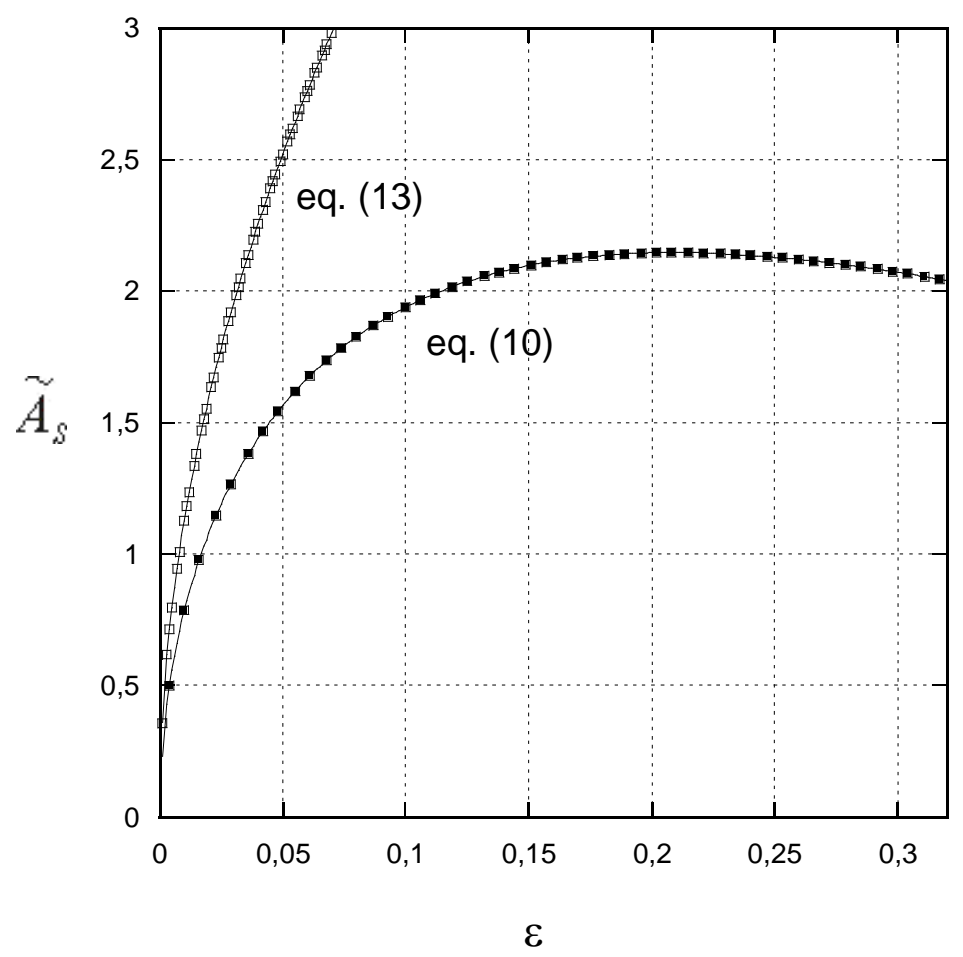

Figure 2: Dimensionless specific surface area as a function of $\varepsilon$ 
From eqs.(2), (4), (5) and (9), the equivalent specific surface area of the foam thus expresses as:

$$
A_{S}=\frac{3}{R}(1-\varepsilon)\left[\frac{s(\varepsilon)}{s_{0}}-\frac{s_{f}(\varepsilon)}{s_{0}}\right]
$$

The dimensionless specific surface area, $\tilde{A}_{s}=R A_{s}$, is plotted in figure 2 as a function of $\varepsilon$. Induced by the strong decrease of the films surface area for increasing liquid volume fractions, $\tilde{A}_{s}$ is an increasing function of $\varepsilon$, despite the reduction in the total interfacial area of the foam. This evolution reverses as the liquid fraction reaches $\varepsilon \approx 0.2$.

It is instructive to compare this result with the one that can be deduced from the classical channels model. In the dry foam limit, it has been early recognized that liquid flow essentially proceeds through the Plateau borders [17], that are the liquid channels emerging from the thin films junctions, three by three, and arranged along the polyedral bubbles edges. One can obtain the mean hydraulic radius of these channels:

$$
m=s_{P b} / p_{P b}=\delta_{a} r / \pi
$$

where $s_{P b}$ and $p_{P b}$ are respectively the cross-sectional area and the perimeter of the Plateau border cross-section, $r$ is the mean radius of curvature for the interfaces, and $\delta_{a}=\sqrt{3}-\pi / 2$. In the dry limit of Kelvin foam, the radius of curvature $r$ is known to depend on $\varepsilon$ and on R, the radius of the volume-equivalent spherical bubble [18]:

$$
r \cong \sqrt{3} R \varepsilon^{1 / 2}
$$


From eqs (2), (11) and (12), the dimensionless specific surface area is deduced:

$$
\tilde{A}_{s} \cong\left(\frac{\pi}{\sqrt{3} \delta_{a}}\right) \varepsilon^{1 / 2}
$$

As a comparison, $\tilde{A}_{S}$ is plotted as a function of $\varepsilon$ in figure 2. As this analysis has been developed within the dry foam approximation, the plot of eq.(13) is limited to the range $\varepsilon \leq 0.07$. From the comparison of the two theoretical curves, it is shown that eq.(13) provides overestimated values for $\tilde{A}_{S}$, even in the dry foam limit where a factor close to 1.5 is obtained. Obviously, this large deviation has to be attributed to the fact that, within the framework of the channels model, the connectivity of the network is ignored. In other words, all the liquid is assumed to be confined within the channels whereas some amount is contained within the channels junctions. The precise description of the foam geometry on the micro-scale, in terms of respective volumes of the channels and their junctions, is not an easy task and has been the subject of several studies [3,5].

Using eqs (3) and (10), the permeability of the Kelvin foam is obtained and the dimensionless permeability $k / D_{b}^{2}$ (with the bubble diameter $D_{b}=2 R$ ) is plotted in figure 3 as a function of $\varepsilon$. The value of $C_{K}$ is set equal to 5 . 


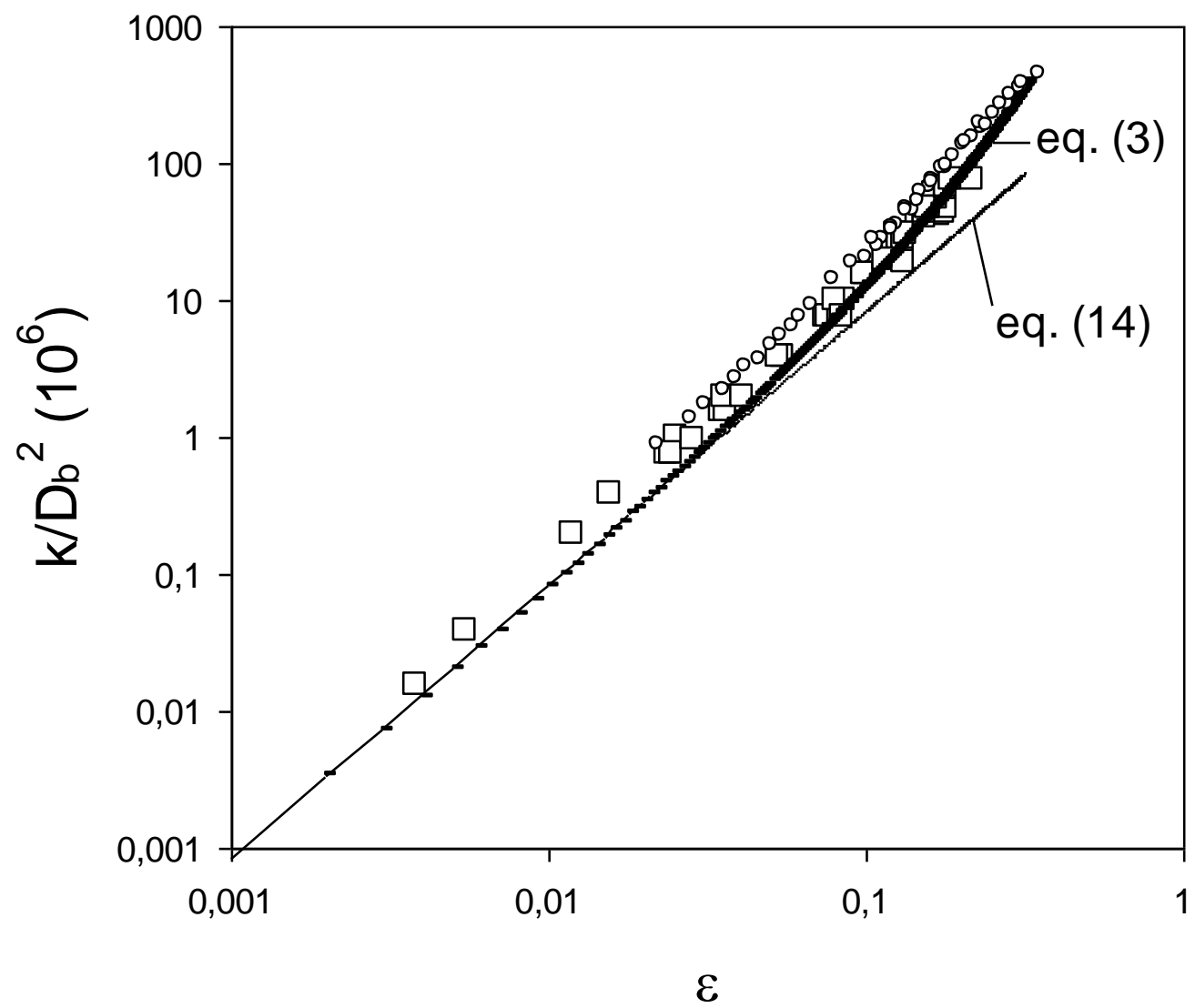

Figure 3: Dimensionless foam permeability as a function of $\varepsilon$. Experimental data (open squares) are plotted against available theory for dry foams (eq. 14) and the theory derived in this paper. Open circles correspond to data published in [7] for oil-in-water emulsions.

\section{EXPERIMENTS}

We measured foam permeability during steady gravity drainage experiments. To obtain the foam, we release into the foaming solutions a slow flow of $\mathrm{C}_{6} \mathrm{~F}_{14}$ gas either through a bluntend syringe needle or through a porous glass frit. The foam shows no sign of coarsening on day-time. To measure the diameter of the bubbles we sample tens of bubbles, squeeze them between two glass plates separated from $100 \mu \mathrm{m}$ and measure the surface exposed with a microscope. Using volume conservation, we calculate the volume of gas inside the bubble and the diameter $D_{b}$ of the bubble. This method ensures a very good precision over the 
measurement of the bubbles diameter. Typically, the standard deviation of the bubbles distribution is less than $4 \%$ when the foam is produced with a needle. The foam obtained from the porous glass frit is less regular with a typical standard deviation of $18 \%$.

To perform the drainage experiment, the foam is put inside a Perspex tube. We use two different cylindrical tubes respectively 50 and $60 \mathrm{~cm}$ long with a section $\sigma$ of 22.90 and 8.81 $\mathrm{cm}^{2}$ cross section areas. We make sure that the section of the tubes contains at least 20 bubbles. The bottom of the tube is in contact with the foaming solution and the top of the tube is open.

The foaming solution is composed of distilled water and surfactants. We used TTAB (TetradecylTrimethylAmmonium Bromide) at $3 \mathrm{~g} / \mathrm{L}$ mixed with dodecanol at concentration $0.2 \mathrm{~g} / \mathrm{L}$. This formulation ensures low mobility conditions for the fluid interfaces $[19,20]$, so that the resulting liquid velocity profile is expected to be a good approximation of a Poiseuille-type flow. All the surfactants were purchased from Aldrich and used as received. The foaming solution has a surface tension $\gamma=38 \pm 1 \mathrm{mN} / \mathrm{m}$, a viscosity $\mu=1 \mathrm{mPa}$.s and a density $\rho=1000 \mathrm{~kg} / \mathrm{m}^{3}$.

Prior to any measurement, we wait for twenty minutes that the foam inside the solution has reached static equilibrium. This ensures a very dry, well-drained foam with no sign of evolution. This equilibrium being reached, a constant flux of the soap solution $\mathrm{Q}$ is added at the top of the foaming tube. Typically, $\mathrm{Q}$ ranges from 0.1 to $50 \mathrm{~mL} / \mathrm{min}$. The foam is uniformly illuminated from one side and a digital camera with a field of view of $20 \mathrm{~cm}$ by 10 $\mathrm{cm}$ records the transmitted light at a frame rate of 10 images/second. Wet foams transmit less light than dry foams, so the camera easily detects the liquid velocity $\mathrm{v}_{1}$ in the laboratory frame. The bottom of the foam column - in contact with the foaming solution - is static, thus, as liquid is introduced in the top of the column, the foam expands and the bubbles move up. We measure the ascending velocity of bubbles $U$ and we deduce the front velocity $v_{f}-$ that is the 
liquid velocity in the frame of the bubbles - from $\mathrm{v}_{\mathrm{l}}$ and $\mathrm{U}$ using $\mathrm{v}_{\mathrm{f}}=\mathrm{v}_{\mathrm{l}}+\mathrm{U}$. We also make sure that the upward motion of the foam is indeed due to expansion as liquid is introduced at the top of the column checking that $\mathrm{U}=\mathrm{Q} / \sigma$.

We determined the foam permeability: $k=(\mu / \rho g)(Q / \sigma)$ as a function of the liquid fraction $\varepsilon=Q / \sigma v_{f}$. The dimensionless permeability $k / D_{b}^{2}$ is plotted in figure 3 as a function of $\varepsilon$.

Our data are in very good aggreement with published experimental results for surfactants foams with low-mobility interfaces, such as proteins foams [6].

\section{DISCUSSION}

Figure 3 shows the comparison of experimental results for the permeability of a foam with non-mobile interface conditions and the theoretical estimation from the Carman-Kozeny model. Surprisingly the agreement is excellent, in spite of the fact that no attempt has been done to adjust the value of $C_{K}$. Figure 3 emphasizes the relevance of the Carman-Kozeny model, that predicts the foam permeability over two orders of magnitude for the liquid fraction. Obviously, this agreement can be attributed to the fact that the liquid flow through the foam is confined inside a network of connected channels, that are the primary elements of the Carman-Kozeny model. As an additional reason, it can be mentioned that, due to the fluid nature of interfaces, the geometry of the channels is dictated by the Laplace law, ensuring a constant channel pore size and a constant network connectivity within each cross-section throughout the sample.

Considering the viscous dissipation in the network of long and slender Plateau Border channels, models for dry foams permeability have been proposed. Assuming a Poiseuille flow and non-mobile interfaces, the foam permeability was proposed to be given by [2]: 


$$
k_{c}=3.210^{-3} R^{2} \varepsilon^{2}
$$

The corresponding dimensionless permeability is plotted in figure 3. Values provided by the two models are very close within the range $\varepsilon \leq 0.05$. In fact, within this range, both models successfully compare with experimental results. However, as liquid fraction increases, increasing deviations are observed: for example, $\mathrm{k} / \mathrm{k}_{\mathrm{c}} \approx 1.27$ for $\varepsilon=0.06$, and $\mathrm{k} / \mathrm{k}_{\mathrm{c}} \approx 4.51$ for $\varepsilon$ $=0.32$. As expected, these results confirm that eq.(14) fails in predicting the permeability of wet foams, i.e. for $\varepsilon \geq 0.05$.

Finally, we compare the present result established for foams with results available for emulsions. In a recent work, Peron et al. [7] addressed the issue of drainage by evaluating the flow conductivity of emulsions and linked it to the variation of electrical conductivity with liquid fraction. We use these published experimental values and we plot the corresponding permeability values in figure 3 . These data compare well with those for foams, although slightly above. The observed deviation can be attributed to strong container wall effect [7] or to the deviation from the no-slip assumption (Poiseuille) for the flow with this system, in spite of the relatively high viscosity of the dispersed phase. This comparison thus suggests that the proposed model can be used to describe the permeability of emulsions, and appears to be an alternative theoretical tool as soon as non-mobile interfaces are concerned.

\section{CONCLUSIONS:}

We have determined an equivalent specific surface area for a liquid foam with Kelvin structure. Then, using the Carman-Kozeny model originally developed for granular beds, the foam permeability has been estimated. We performed measurements for the permeability of liquid foams with non-mobile interface conditions, and we have shown that these experimental data are accurately described by this model over two decades for the foam liquid 
fraction, without any adjustable parameter. To our knowledge, this result constitutes the strongest link relating liquid foams to solid porous media.

ACKNOWLEDGMENT: We thank Pierre Mills and Xavier Chateau for fruitful discussions.

We acknowledge the Agence Nationale de la Recherche (ANR-05-JCJC-0234-01), the European Space Agency (MAP "Hydrodynamics of wet foams") and the french space agency (C. N. E. S.) for fundings.

\section{REFERENCES:}

[1] Darcy H., Les fontaines publiques de la ville de Dijon.; V. Damont: Paris, 1856.

[2] Weaire D., Pittet N., Hutzler S. and Pardal D., Phys. Rev. Let. 1993, 71, 2670.

[3] Koehler S. A., Hilgenfeldt S. and Stone H. A., Langmuir 2000, 16, 6327.

[4] Carrier V., Destouesse S. and Colin A., Phys. Rev. E, 2002, 65, 061404.

[5] Neethling S. J., Lee H. T. and Cilliers J. J., J. Phys.: Condens. Matter 2002, 14, 331.

[6] Saint-Jalmes A., Zhang Y. and Langevin D., Eur. Phys. J. E 2004, 15, 53.

[7] Peron N., Cox S.J., Hutzler S., and Weaire D., Eur. Phys. J. E 2007, 22, 341-351.

[8] Kozeny, Ber. Wien Akad. 1927, 136a, 271.

[9] Carman P.C., Trans. Inst. Chem. Eng. 1937, 15, 150.

[10] Coelho D., Thovert J.-F. and Adler P.M., Phys. Rev. E 1997, 55 (2), 1959-1978.

[11] Mourzenko V., Thovert J.-F., Vizika O. and Adler P.M., Phys. Rev. E 2008, 77, 066306.

[12] Dullien F. A. L., Porous Media. Fluid Transport and Pore Structure.; Academic: New York, 1992

[13] Brakke K., Exp. Math. 1992, 1, 141.

[14] Lacasse M.-D., Grest G. S. and Levine D., Phys. Rev. E 1996, 54 (5), 5436. 
[15] Höhler R., Yip Cheung Sang Y., Lorenceau E. and Cohen-Addad S., Langmuir 2008, 24, 418-425.

[16] Hilgenfeldt S., Koehler S.A. and Stone H.A., Phys. Rev. Lett. 2001, 86, 4704.

[17] Leonard R.A. and Lemlich R. AIChE J. 1965, 11, 18.

[18] Weaire D., Hutzler S., The Physics of Foams; Oxford University Press: Oxford, 1999.

[19] Pitois O., Fritz C. and Vignes-Adler M., Coll. Surf. A 2005, 261, 109-114.

[20] Pitois O., Fritz C. and Vignes-Adler M., J. Colloid Interface Sci. 2005, 282, 458-465. 